\title{
The effect of low dose aspirin and low molecular weight heparin (enoxaparin) in recurrent pregnancy loss associated with antiphospholipid antibody syndrome
}

\author{
Sasmita Swain, Sujata Singh*
}

Department of Obstetrics and Gynecology, S.C.B. Medical College and Hospital, Cuttack, Odisha, India

Received: 08 September 2017

Accepted: 28 September 2017

*Correspondence:

Dr. Sujata Singh,

E-mail: sujatamuniraj@gmail.com

Copyright: () the author(s), publisher and licensee Medip Academy. This is an open-access article distributed under the terms of the Creative Commons Attribution Non-Commercial License, which permits unrestricted non-commercial use, distribution, and reproduction in any medium, provided the original work is properly cited.

\begin{abstract}
Background: Recurrent miscarriage affects 1-2\% of women. Recurrent pregnancy loss (RPL) is the loss of three or more consecutive pregnancies before or during the 20th week of gestation. The most important association between gestational loss and autoimmune phenomena is the presence of antiphospholipid antibodies represented by the lupus anticoagulants and or anticardiolipin antibodies (Antiphospholipid Antibody Syndrome). The antiphospholipid syndrome is an acquired autoimmune. The clinical features are thrombosis (venous, arterial and microvascular) and/or pregnancy complications; the most prominent of which is recurrent abortion.

Methods: Twenty-two selected patients during pregnancy with clinical and/or serological findings of antiphospholipid syndrome had received low dose aspirin (75 mg once daily orally) plus LMWH enoxaparin (40 mg subcutaneously/day).

Results: There are live born in $86 \%$ cases compared to abortion ( $<20$ weeks gestational age) in $14 \%$ cases. From 19 liveborn babies the mother having normotensive in $79 \%$ and preeclampsia $21 \%, 85 \%$ babies having normal growth and $15 \%$ are IUGR. $36 \%$ cases are at term ( $>37$ weeks) and $50 \%$ cases are at preterm (<37 week) on which $9 \%)$ is spontaneous preterm and $41 \%$ is iatrogenic preterm due to preeclampsia, IUGR, PPROM and APH.

Conclusions: Use of low dose aspirin (75mg) and enoxaparin $40 \mathrm{mg}$ subcutaneously daily in patients with RPL due to antiphospholipid syndrome resulted in higher live birth rates. Combination treatment with aspirin and LMWH leads to a high live birth rate among women with recurrent abortion and antiphospholipid antibodies.
\end{abstract}

Keywords: aPL, APS, APA, IUGR, LDA, LMWA, RPL

\section{INTRODUCTION}

The antiphospholipid syndrome (APS) is a systemic autoimmune disease characterized by arterial and venous thrombosis, gestational morbidity and presence of elevated and persistently positive serum titres of antiphospholipid antibodies. It is currently recognized as the most frequent cause of acquired thrombophilia associated with venous and arterial thrombosis. Nowadays, APS is recognized as the most significant cause of RPL (recurrent pregnancy loss). Thrombophilia included antiphospholipid syndrome has been identified in about $50 \%$ of women with recurrent miscarriage. Up to 15- $20 \%$ women with recurrent pregnancy loss have antiphospholipid antibodies (aPL).

Women with antiphospholipid syndrome (APS) have a spontaneous abortion rate as high as $90 \%$ for pregnancies without pharmacologic treatment. ${ }^{1}$ APS is strongly associated with recurrent abortion and pregnancy complications such as intrauterine growth restriction (IUGR), preterm labor, preeclampsia, and intrauterine 
foetal death (IUFD). The adverse effects of antiphospholipid antibodies on trophoblast differentiation and invasion, placental infarctions, and thrombosis are thought to be responsible for recurrent abortion and pregnancy complications associated with APS. ${ }^{2,3}$

Antiphospholipid antibodies can be detected in $1-5 \%$ of healthy women. The prevalence of positive antiphospholipid antibodies increases to $15 \%$ in women with recurrent first trimester pregnancy losses and up to $20 \%$ in women suffering a stroke at or before the age of 50 years. Around $40 \%$ of women with lupus have antiphospholipid antibodies; it is estimated that less than $40 \%$ of them will eventually develop thrombotic events. The prevalence of APS is unknown, but it has been estimated to be $0.5 \%$ in the general population. ${ }^{4}$

There are three primary classes of antibodies associated with the antiphospholipid syndrome:

- $\quad$ anticardiolipin antibodies (aCL),

- the lupus anticoagulant (LA) and

- antibodies directed against specific molecules including a molecule known as beta-2-glycoprotein 1 (anti-b2GPI).

Lupus antibody is the most powerful predictor of thrombosis and recurrent miscarriages. Anti-b2glycoprotein antibodies are not associated with recurrent miscarriage in isolation; however, in combination with positive results for lupus anticoagulant (LA) and aCL, there is a high risk of obstetric complications. ${ }^{5}$

The current classification (Revised Sapporo Criteria for diagnosis of definite APS) meant for inclusion in clinical research protocols, but often used in daily practice to establish the diagnosis of APS and indicate a treatment, was reviewed in 2006 and includes clinical and laboratory criteria. ${ }^{6}$

\section{Clinical criteria}

- Vascular thrombosis:

one or more episodes of arterial or venous thrombosis or thrombosis of small vessels of any organ or tissue, confirmed on Doppler or histopathology, vasculitis excluded;

- Gestational morbidity:

a) One or more deaths of a morphologically normal foetus after the 10th gestational week, confirmed on ultrasound or by examining the foetus;

b) One or more premature births of a morphologically normal foetus before the 34th gestational week due to eclampsia, preeclampsia or causes of placental insufficiency;

c) Three or more spontaneous abortions before the $10^{\text {th }}$ gestational week, with neither maternal hormonal nor anatomical abnormalities, paternal and maternal chromosomal causes excluded.

\section{Laboratory criteria}

- Presence of lupus anticoagulant antibody (LA) in the plasma on two or more occasions at a minimum 12week interval, detected according to the recommendations of the International Society on Thrombosis and Haemostasis (ISTH);

- Moderate (> 40) to high (> 80) titres of IgG or IgM anticardiolipin antibodies (ACL) on two or more occasions at a minimum 12-week interval, detected by using standard ELISA test;

- $\quad$ IgG or IgM anti-beta 2-GPI antibodies in the plasma on two or more occasions at a minimum 12-week interval, detected by using standard ELISA test.

Clinical manifestations may range from no symptoms to immediately life threatening catastrophic APS. ${ }^{6}$ Primary APS is defined as presence of aPL anti bodies in patient with idiopathic thrombosis but no evidence of autoimmune disease. Secondary APS is used when patients with a wide spectrum of autoimmune disorders (primarily SLE and rheumatoid arthritis) and thrombosis are also found to have antiphospholipid antibodies. ${ }^{7}$ Probable APS is one in which there are typical clinical manifestations but without positive serological test of aPL. These are also called seronegative APS or pre-APS. ${ }^{8}$

Without treatment, the miscarriage rate in a subsequent pregnancy in this condition is as high as $90 \%$. It is widely accepted that treatment with low dose aspirin and heparin or low molecular weight heparin (LMWH) significantly improve out come as compared to previous untreated pregnancies. ${ }^{9}$

During the past 27 years, plentiful treatments such as unfractionated heparin (UFH), low molecular weight heparin (LMWH), plasmapheresis, moderate-to-high dose prednisone, intravenous immunoglobulin, and low-dose aspirin (LDA) have been used in the management of pregnant Women with history of recurrent pregnancy loss secondary to APS. ${ }^{2}$ A meta-analysis of 13 randomized or quasi-randomized controlled trials of various management options for pregnant women with a history of recurrent abortion secondary to APS found that combined UFH and LDA was the best management option, reducing pregnancy loss by $54 \% .^{3,10-12}$ A number of studies have evaluated the efficacy of treatment with low-dose aspirin, prednisolone, unfractionated lowmolecular weight heparin and most recently intravenous gamma globulin, either alone or in various combinations. However, the findings have not been consistent. ${ }^{13,14}$ Lowdose aspirin in combination with heparin was demonstrated in two randomized controlled trials to lead to a significant improvement in the live birth rate. ${ }^{15,16}$ This study aimed to determine the pregnancy outcome in women with APS and recurrent pregnancy loss who were treated with aspirin alone or aspirin in combination with heparin during the index pregnancy. 
Use of low dose of aspirin and low molecular weight heparin (Enoxaparin) is safe in pregnancy and it improves foetal outcome. ${ }^{17}$ Bleeding is a potential complication of anticoagulant therapy, heparin induced thrombocytopenia has been observed less commonly in patients treated with LMWH. LMWH do not cross the placenta and therefore are not associated with bleeding in foetuses and have no teratogenic effects. ${ }^{10}$ LMWH have higher specificity for $\mathrm{Xa}$ and have fewer effects on platelet activity. As a result, LMWH may cause bleeding less often, while still retaining anticoagulant effects. The LMWH are associated with less risk of heparin induced osteoporosis.

\section{METHODS}

The study of low dose aspirin and LMWH in recurrent pregnancy loss associated with APS was carried out in the department of $\mathrm{O}$ and $\mathrm{G}$ in S.C.B Medical College, Cuttack during period of September 2014 to November 2016.

Cases were selected from OBGYN OPD in the age group between 19- 37 years with two or more consecutive first trimester or second trimester pregnancy loss with positive lupus anticoagulant or anticardiolipin antibody IgG ACA $>10 \mathrm{GPL}$ or IgM > $10 \mathrm{MPL}$ on at least two occasions 6 to 12 weeks apart excluding Anatomical abnormality, parenteral chromosomal abnormality, luteal phase defect, endocrinological abnormality by thorough investigations. In this study, mostly anticardiolipin antibody was tested. Thyroid abnormality and hyperprolactinemia and Diabetes mellitus were also excluded from the study. The present study was conducted on total of 22 patients.

Patients were advised to take aspirin $75 \mathrm{mg}$ orally prior to conception. Each pregnancy was documented by transvaginal USG scheduled at 6-7-week gestation for the determination of foetal heart activity. After confirmation of foetal cardiac activity inj. Enoxaparin $40 \mathrm{mg}$ s.c. daily started and continued up to 34 weeks. For some patients aspirin and enoxaparin were discontinued earlier due to abortion, preterm labour, preeclampsia, leading to premature delivery. Baseline investigation i.e. complete blood count, urine routine examination, blood sugar, grouping, Rh typing, BT, CT, PT, aPTT, Sr. creatinine concentration, urine protein creatinine ratio, $\mathrm{Sr}$. ALT/AST. HIV, HBSAg, HCV screening were offered to all patients and findings noted as soon as they conceived. Folic acid $400 \mathrm{mcg}$ was started preconceptionally till 12 weeks. of gestation and iron and calcium started which was continued till 6-month post-partum. Patient has undergone ANC at every 2-4 week interval upto 20 week and every 1-2 week thereafter. The objective of prenatal care was to monitor for maternal hypertension, proteinuria and other feature of preeclampsia. Obstetric USG at 3-4 week interval beginning at 17-20 week done to assess foetal growth and amniotic fluid volume. The timing of delivery was assessed individually on the basis of current and past obstetric history and the onset of complications e.g. preeclampsia, IUGR or abnormal
Doppler result. Women whose pregnancy reached 38 completed weeks of gestation were either induced or delivered by elective Caesarean section.

\section{RESULTS}

It is observed that out of 22 patients 19 patients (86\%) delivered a live baby after treatment with low dose aspirin and LMWH, but there is abortion of 3 patients $(14 \%)$ even after the medication (Table 1).

Table 1: Pregnancy outcome.

\begin{tabular}{|lll|}
\hline Outcome & No. of cases & Percentage \\
\hline Live born & 19 & 86 \\
\hline Abortion & 3 & 14 \\
\hline
\end{tabular}

It is observed that in spite of treatment with low dose aspirin and LMWH the incidence of preeclampsia is around $21 \%$ of APS (Table 2 ).

Table 2: Incidence of preeclampsia.

\begin{tabular}{|lll|}
\hline PIH & No. of cases & Percentage \\
\hline Normotensive & 15 & 79 \\
\hline Preeclampsia & 4 & 21 \\
\hline
\end{tabular}

The table shows that incidence of IUGR is $15 \%$ after with low dose aspirin and LMWH in APS (Table 3).

Table 3: Incidence of IUGR.

\begin{tabular}{|lll|}
\hline & No. of cases & Percentage \\
\hline IUGR & 3 & 15 \\
\hline Normal growth & 16 & 85 \\
\hline
\end{tabular}

Table shows that only $36 \%$ of cases delivered at term. 2 cases $(9 \%)$ had spontaneous preterm labour and 9 cases delivered preterm for preeclampsia, IUGR, PROM (Table 4).

Table 4: Gestational age at time of delivery.

\begin{tabular}{|ll|l|}
\hline Gestational age & No. of cases & Percentage \\
\hline$>37$ week (term) & 8 & 36 \\
\hline $\begin{array}{l}<37 \text { week (prerterm) } \\
\text { spontaneous }\end{array}$ & 2 & 9 \\
$\begin{array}{l}<37 \text { week (prerterm) } \\
\text { iatrogenic }\end{array}$ & 9 & 41 \\
\hline Spontanous abortion & 3 & 14 \\
\hline
\end{tabular}

Table shows that $95 \%$ of cases delivered by caesarean section and only 1 case had vaginal delivery (Table 5).

Table 5: Mode of delivery.

\begin{tabular}{|lll|}
\hline Route & No. cases & Percentage \\
\hline LSCS & 18 & 95 \\
\hline VD & 1 & 5 \\
\hline
\end{tabular}




\section{DISCUSSION}

In this study, it was found maximum no. of cases were in age group of 20-30 year. In the present series, maximum no. of women with history of recurrent pregnancy loss was found to belong $\mathrm{G}_{3}$ and $\mathrm{G}_{4}$. The risk of subsequent pregnancy loss in woman with antiphospholipid antibody and previous pregnancy loss is as high as $90 \%$ (Rai et al), which occurs between 7 and 12 weeks of gestation and represent the loss of chromosomally normal foetus. ${ }^{1}$ In this present study, live birth rate is $86 \%$ after medication. In spite of treatment 3 out of 22 cases (14\%) were pregnancy loss. In a study by Fawad $\mathrm{S}$ it was observed 93\% live births and 7\% early pregnancy loss in spite of treatment with low dose aspirin and LMWH. ${ }^{18}$ In a study by David MO it was found to be $80 \%$ live births and $20 \%$ miscarriage in treatment of low dose enoxaparin with low dose aspirin. ${ }^{19}$ Study by Stephenson MD compared daltiparine to unfractionated heparin observed $69 \%$ women have successful pregnancy in daltiparine. ${ }^{17}$ According to Dadhwal V et al at AIIMS reported live birth rate of $85 \%$ with treatment with low dose aspirin and Heparin. ${ }^{20}$ In another study by Farguharson RG et al observed $72 \%$ success when low dosed aspirin used for APS in pregnancy. ${ }^{21}$

In the present study $21 \%$ developed preeclampsia despite treated with low dose aspirin and LMWH. Study by Katarina Jeremic et al shows that preeclampsia occurs $15 \%$ despite treatment with low dose aspirin and LMWH. ${ }^{22}$ In a study by Fawad $\mathrm{S}$ it was observed only $7 \%$ patients develop preeclampsia despite treated with low dose aspirin and LMWH. ${ }^{18}$ In Portugal, a study by Sernano et al shows incidence of preeclampsia $19.4 \%$ with medication. ${ }^{23}$

In the present study only $36 \%$ of cases delivered at term. 2 cases $(9 \%)$ had spontaneous preterm labour and 9 cases (41\%) delivered preterm for preeclampsia, IUGR, PROM. In a study by Brenner et al rate of preterm delivery was $9 \%$ which is comparable to present study. ${ }^{24}$ In another study by David MO et al 5\% underwent preterm delivery despite treated with low dose aspirin and LMWH. ${ }^{19}$

In present study incidence of IUGR is $15 \%$ after treatment with low dose aspirin and LMWH in ACA antibody positive patient. In study by Clark $\mathrm{P}$ et al there was increase risk of IUGR. ${ }^{25}$ In another study by Glasnovices et al observed $8.3 \%$ cases of IUGR after treatment with low dose aspirin and LMWH. ${ }^{26}$ In a study by Backos $\mathrm{M}$ et al observed $15 \%$ of infants were small for gestational age when treated with LDA and heparin. ${ }^{27}$

In present study $95 \%$ cases were delivered by LSCS and only 1 case $(5 \%)$ was delivered vaginally. Saadia Fawad observed $84 \%$ cases by LSCS and $16 \%$ by vaginally despite treatment with low dose aspirin and LMWH. ${ }^{18}$ In a study by Serrano $\mathrm{F}$ et al, $54.4 \%$ cases delivered by LSCS. $^{23}$ studies confirmed that treatment with LMWH plus aspirin should be considered as the standard therapy for recurrent pregnancy loss due to APS. ${ }^{11,28}$

Combination treatment with aspirin and LMWH leads to a high live birth rate among women with recurrent miscarriage and antiphospholipid antibodies. This combination may promote successful embryonic implantation in the early stages of pregnancy and protect against thrombosis of the uteroplacental vasculature after successful placentation which leads to decrease percentage of IUGR and PIH.

\section{ACKNOWLEDGMENTS}

The authors thank Prof. Dr. Lucy Das HOD Department of $\mathrm{O}$ and $\mathrm{G}$ in S.C.B Medical College and Hospital for her valuable collaboration in this project.

Funding: No funding sources

Conflict of interest: None declared

Ethical approval: The study was approved by the Institutional Ethics Committee

\section{REFERENCES}

1. Rai RS, Clifford K, Cohen H, Regan L. High prospective fetal loss rate in untreated pregnancies of women with recurrent miscarriage and antiphospholipid antibodies. Hum Reprod. 1995;10(12):3301-4.

2. Tuthill JI, Khamashta MA. Management of antiphospholipid syndrome. J Autoimmun 2009;33(2):92-8.

3. Empson M, Lassere M, Craig J, Scott J. Prevention of recurrent miscarriage for women with antiphospholipid antibody or lupus anticoagulant. Cochrane Database Syst Rev. 2005;18(2):CD002859.

4. Cohen D, Berger SP, Steup-Beekman GM, Bloemenkamp KW, Bajema IM. Diagnosis and management of the antiphospholipid syndrome. BMJ 2010;340:1125-32.

5. Galarza-Maldonado C, Kourilovitch MR, PérezFernández OM, Gaybor M, Cordero C, Cabrera S et al. Obstetric antiphospholipid syndrome. Autoimmunity Rev. 2012 Feb 29;11(4):288-95.

6. Miyakis S, Lockshin MD, Atsumi T, Branch DW, Brey RL, Cervera $\mathrm{R}$ et al. International consensus statement on an update of the classification criteria for definite antiphospholipid syndrome (APS). J Thrombosis Haemostasis. 2006 Feb 1;4(2):295-306.

7. Baker Jr WF, Bick RL. The clinical spectrum of Antiphospholipid syndrome. Hematol Oncol Clin N Am. 2008;22:35-52.

8. Hughes GR, Khamashta MA. Seronegative anti phospholipid syndrome. Ann Rheum Dis. 2003;62:1127.

9. Berg TG. Antiphospholipid antibody syndrome and pregnancy. 2009. Available at 
http://emedicine.Medscape.Com/article/261691print.

10. Bates SM, Greer IA, Hirsh J, Ginsberg JS. Use of antithrombotic agents during pregnancy: the seventh ACCP Conference on antithrombotic and thrombolytic therapy. Chest 2004;126(3 Suppl):627S-44S.

11. Triolo G, Ferrante A, Ciccia F, Accardo-Palumbo A, Perino A, Castelli A, et al. Randomized study of subcutaneous low molecular weight heparin plus aspirin versus intravenous immunoglobulin in the treatment of recurrent fetal loss associated with antiphospholipid antibodies. Arthritis Rheum. 2003;48(3):728-31.

12. Dendrinos S, Sakkas E, Makrakis E. Low-molecularweight heparin versus intravenous immunoglobulin for recurrent abortion associated with antiphospholipid antibody syndrome. Int $\mathbf{J}$ Gynecol Obstet. 2009;104(3):223-5.

13. Stone S, Poston L. Antiphospholipid antibody syndrome in pregnancy: onset to outcome. Foetal Maternal Med Rev. 2004;15:273-97.

14. Empson M, Lassere M, Craig JC, Scott JR. Recurrent pregnancy loss with antiphospholipid antibody: a systemic review of therapeutic trials. Obstet Gynecol 2002;99:135-44.

15. Kutteh WH. Antiphospholipid antibody associated recurrent pregnancy loss: treatment with heparin and low dose aspirin is superior to low dose aspirin alone. Am J Obstet Gynecol. 1996;174:1584-9.

16. Rai R, Cohen H, Dave M, Regan L. Randomised controlled trial of aspirin plus heparin in pregnant women with recurrent miscarriage associated with antiphospholipid antibodies. $\mathrm{Br}$ Med J. 1997;314:253-7.

17. Stephenson MD, Ballem PJ, T sang P, Purkiss S, Houlihan E, Enson MH. Treatment of antiphospholipid antibody syndrome (APS) in pregnancy: a random pilot total comparing low molecular weight Heparin to unfractionated Heparin. J Obstet Gynacol Can. 2004;26:729-34.

18. Saadia Fawad Dept. of Gynecology, Aero Hospital. Role of antithrombotic therapy for recurrent pregnancy loss due to APS. J Ayub Med Coll Abbottabad. 2010;22(3).

19. Mo D, Saravelos S, Metwally M, Makris M, Li TC. Treatment of recurrent miscarriage and antiphospholipid syndrome with low-dose enoxaparin and aspirin. Reprod Biomed Online. 2009 Jan 1;19(2):216-20.

20. Dadhwal V, Sharma AK, Deka D, Gupta B, Mittal S. The obstetric outcome following treatment in a cohort of patients with antiphospholipid antibody syndrome in a tertiary care center. J Postgraduate Med. 2011 Jan 1;57(1):16.

21. Farquharson RG, Quenby S, Greaves $M$. Antiphospholipid syndrome in pregnancy: a randomized, controlled trial of treatment. Obstet Gynecol. 2002;100:408-13.

22. Jeremić K, Pervulov M, Gojnić M, Dukanac J, Ljubić A, Stojnić J. Comparison of two therapeutic protocols in patients with antiphospholipid antibodies and recurrent miscariages. Vojnosanitetski Pregled. 2005;62(6):435-9.

23. Serrano F, Nogueira I, Borges A, Branco J. Primary antiphospholipid syndrome: pregnancy outcome in a portuguese population. Acta Reumatologica Portuguesa. 2009;34(3):492-7.

24. Brenner B, Hoffman R, Blumenfeld Z, Weiner Z, Younis JS. Gestational outcome in thrombophilic women with recurrent pregnancy loss treated by enoxaparin. Thrombosis Haemostasis. 2000;83(5):693-7.

25. Clark P, Walker ID, Langhorne P, Crichton L, Thomson A, Greaves M, et al. Scottish Pregnancy Intervention Study (SPIN) Collaborators. SPIN (Scottish Pregnancy Intervention) study: a multicenter, randomized controlled trial of lowmolecular-weight heparin and lowdose aspirin in women with recurrent miscarriage. Blood. 2010;115:4162-7.

26. Glasnovic M, Bosnjak I, Vcev A, Soldo I, Kosuta M. Antibody profile of pregnant woman with APS and Pregnancy outcome after treatment with LDA and LMWH. Coll Antropl. 2007;31(1):173-7.

27. Backos M, Rai R, Baxter N, Chilcott IT, Cohen H, Regan L. Pregnancy complications in women with recurrent miscarriage associated with antiphospholipid antibodies treated with low dose aspirin and heparin. BJOG: Int J Obstet Gynecol. 1999 Feb 1;106(2):102-7.

28. Royal College of Obstetrician and Gynaecologists. The investigation and treatment of recurrent miscarriage (internet), Guideline No 17. London: RCOG Press; 2003 May (cited 2009 Feb 8):13.

Cite this article as: Swain S, Singh S. The effect of low dose aspirin and low molecular weight heparin (enoxaparin) in recurrent pregnancy loss associated with antiphospholipid antibody syndrome. Int J Reprod Contracept Obstet Gynecol 2017;6:4830-4 